\title{
Effect of pressure on a defect-related band-resonant vibrational mode in implantation disordered GaAs
}

\author{
T. Sauncy and M. Holtz \\ Department of Physics, Texas Tech University, Lubbock, Texas 79409 \\ R. Zallen \\ Department of Physics, Virginia Tech, Blacksburg, Virginia 24061
}

(Received 11 April 1994)

\begin{abstract}
We have used hydrostatic pressure as a means for studying a resonant Raman mode observed at $47 \mathrm{~cm}^{-1}$ in highly disordered, ion implanted, unannealed GaAs. The mode shifts weakly $(-0.07 \pm$ $\left.0.15 \mathrm{~cm}^{-1} / \mathrm{GPa}\right)$, supporting an identification of this band-resonant vibration as stemming from the breathing mode of the gallium vacancies, which are expected to be in high concentration. We measure a pressure coefficient of the longitudinal-optic phonon in these $(5.5 \mathrm{~nm})$ nanocrystals of $\mathrm{GaAs}$ to be $3.6 \pm 0.1 \mathrm{~cm}^{-1} / \mathrm{GPa}$. The good agreement between our value and the pressure shift of this phonon in bulk GaAs implies that the bulk modulus is independent of size at least down to this size crystallite.
\end{abstract}

\section{INTRODUCTION}

The study of native point defects in semiconductors is of current scientific interest and technological significance, since point defects alter the optical and transport properties of a material. Identifying and studying clear signatures of such defects is an important step in understanding which of these have the most influence on the desired properties and how to control them. What is needed is the identification of spectroscopic signatures produced by particular, known defects.

We study here ${ }^{1}$ one potential identification of a point defect which manifests itself as a resonant-Raman mode in unannealed implanted GaAs. ${ }^{2}$ This resonant, low frequency mode (at $47 \mathrm{~cm}^{-1}$ ) deserves more general notice since it has also been observed in lowtemperature, molecular-beam epitaxially (MBE) grown GaAs. ${ }^{3}$ Furthermore, a very similar Raman mode has been seen in implanted, unannealed silicon. ${ }^{4}$ Recent calculations $^{5}$ of the intrinsic vibrational energy for the $A_{1}$-symmetry (symmetric breathing) mode of the $\mathrm{Ga}$ vacancy $\left(V_{\mathrm{Ga}}\right)$ in $\mathrm{GaAs}$ predict this vibration to be near $40 \mathrm{~cm}^{-1}$. The presence of $V_{\mathrm{Ga}}$ native defects in high concentrations, which is expected in both lowtemperature MBE GaAs and implanted GaAs, offer a plausible identification ${ }^{3}$ of the origin of the resonant mode. The approximate agreement between the calculated and observed low-frequency mode makes $V_{\mathrm{Ga}}$ a good candidate for the microscopic origin of this resonant mode. Furthermore, the observation of a native defect vibrational mode resonant with intrinsic host vibrations is unprecedented.

The application of hydrostatic pressure presents itself as a means by which we can conclusively show that the resonant mode at $47 \mathrm{~cm}^{-1}$ in GaAs stems from a vacancy defect or from disorder activated vibrations of the parent material. The pressure shifts of the intrinsic bulk-
GaAs transverse- and longitudinal-acoustic phonons (TA and LA phonons, respectively), which span the relevant low-vibrational-energy range, are known. ${ }^{6-8}$ On the other hand, we expect a very small pressure shift for the breathing mode of a gallium vacancy. This is because the arsenic atoms participating in the vibration will be influenced little by the spherically symmetric compression: the bonds to the neighboring gallium atoms (where the vibrational energy arises), which make up the secondnearest-neighbor shell to $V_{\mathrm{Ga}}$, will not be substantially deformed. Rather the tetrahedron composed of arsenic atoms which surround the vacancy will take up the contraction.

Raman scattering is proven to be an effective method for studying this class of materials, since it permits measurement of multiple structural phases when simultaneously present. For these measurements, $45 \mathrm{keV}$ beryllium implanted GaAs (no anneal) was chosen for several reasons. The structural properties of the near-surface damaged region have been characterized in detail via Raman scattering. ${ }^{9}$ The high-energy ion implantation, prior to annealing, produces a near-surface damage layer consisting of a mixture of amorphous and nanocrystalline GaAs ( $a$-GaAs and $n$-GaAs, respectively). Most important is the presence of a near-surface region throughout which the damage (average nanocrystallite size and crystallineto-amorphous volume fraction) is uniform. This iondamage layer extends to $\simeq 1500 \AA$. Since the material is opaque in the visible, ${ }^{10,9}$ our Raman probe only measures a macroscopically consistent medium. Second, the resonant conditions necessary to measure the low frequency phonon are easily achieved. Here, hydrostatic pressure is coupled with Raman scattering in an attempt to resolve among the likely interpretations of the observed resonant mode. A second motivation for the work was to observe the effect of pressure on the vibrations native to the $n$ GaAs material. 
We organize this paper as follows. Following a brief description of the experimental setup, we discuss the influence of pressure on the longitudinal-optic (LO) phonon arising from the nanocrystals present in the implanted GaAs. We then discuss our results on the resonant mode and our interpretation of the results as support of the $V_{\mathrm{Ga}}$ breathing-mode picture. Finally, we discuss our findings.

\section{EXPERIMENTAL DETAILS}

The sample used began as a single crystal of (001) GaAs which was implanted with $45 \mathrm{keV} \mathrm{Be}^{+}$ions to a fluence $5 \times 10^{14}$ ions $/ \mathrm{cm}^{2}$, at $7^{\circ}$ off axis, with no post-implantation anneal. Previous studies of materials prepared in this manner show that the ion implantation produces a homogeneous layer approximately 1500 $\AA$ deep, with an average crystallite size of $5.5 \mathrm{~nm} .{ }^{9}$ Since the penetration depth of the Raman probe used is $\frac{1}{2 \alpha}<1000 \AA$ (Ref. 10), only the damaged layer was measured. The samples were back pohshed to a total thickness of approximately 20-30 microns, then cleaved into $\simeq 100 \times 100 \mu \mathrm{m}^{2}$ segments and loaded into a standard diamond anvil cell. ${ }^{11}$ A mixture of 4:1 methanol/ethanol was used as a pressure transmitting medium. Ruby fluorescence was used for pressure calibration. ${ }^{12}$

All Raman spectra were taken at room temperature in direct backscattering geometry. For zero pressure spectra, the sample was placed under an argon atmosphere in order to eliminate $0_{2}$ and $N_{2}$ Raman-active modes. The $647.1 \mathrm{~nm}$ red line from a Krypton laser was used to produce the full-range, first-order vibrational spectra. In order to accomplish these low vibrational energy measurements in the diamond-anvil cell, scattered light was dispersed by a triple-additive-dispersive Raman system which has been described elsewhere. ${ }^{13}$ The $647.1 \mathrm{~nm}$ red line and the argon-ion laser $514.5 \mathrm{~nm}$ green line were each used to obtain spectra in the higher energy range, i.e., to observe the LO phonon of the $n$-GaAs. A multichannel detection system ${ }^{14}$ was also used to obtain measurements of the LO phonon when under pressure.

\section{THE EFFECT OF PRESSURE ON THE LO PHONON IN GaAs NANOCRYSTALS}

In Fig. 1 we show three Raman spectra at various pressures, each covering the first-order vibrational energy range. The characteristic features of Raman spectra from implanted GaAs are seen for all pressures. The LO phonon of the $n$-GaAs is seen at $289 \mathrm{~cm}^{-1}$ at zero pressure. The LO phonon bands are slightly broadened and downshifted when compared to the same features in bulk GaAs. This is a result of the finite size of the crystallites being probed. ${ }^{15}$ The underlying spectrum arises from the amorphous material component of the implanted GaAs. The three broad bands which span the full range of each spectrum are consistent with Raman measurements of amorphous GaAs prepared by conventional means. ${ }^{16}$ The resonant mode at $47 \mathrm{~cm}^{-1}$ is also observable in each spec-

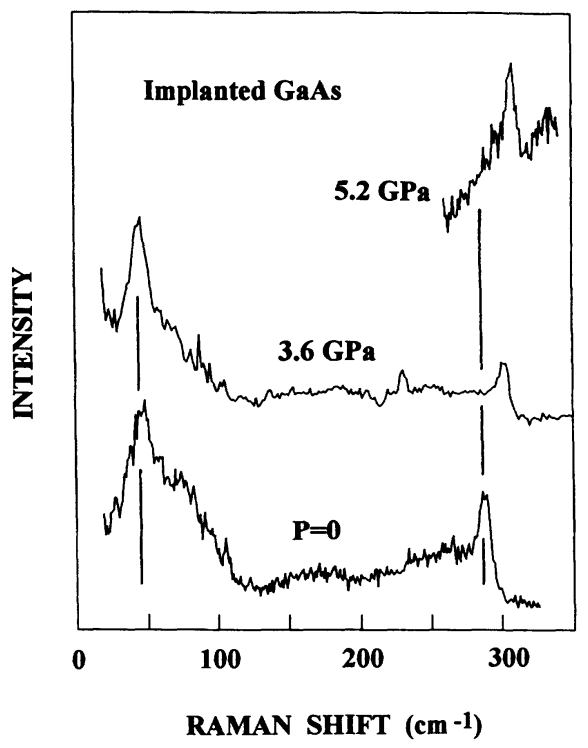

FIG. 1. Room-temperature (Stokes) Raman spectra of 45 $\mathrm{keV} \mathrm{Be}^{+}$implanted GaAs at three different pressures. The LO phonon near $289 \mathrm{~cm}^{-1}$ (vertical line) at zero pressure is seen to shift rapidly with pressure. The feature near $47 \mathrm{~cm}^{-1}$ does not shift substantially in energy to $4 \mathrm{GPa}$.

trum over this pressure range. We discuss this feature in the next section.

The LO phonon upshifts in energy with increasing pressure. The vibrational energy of the LO phonon versus pressure is shown in Fig. 2, together with a linear least squares fit. The best fit yields a pressure dependence for the LO phonon given by

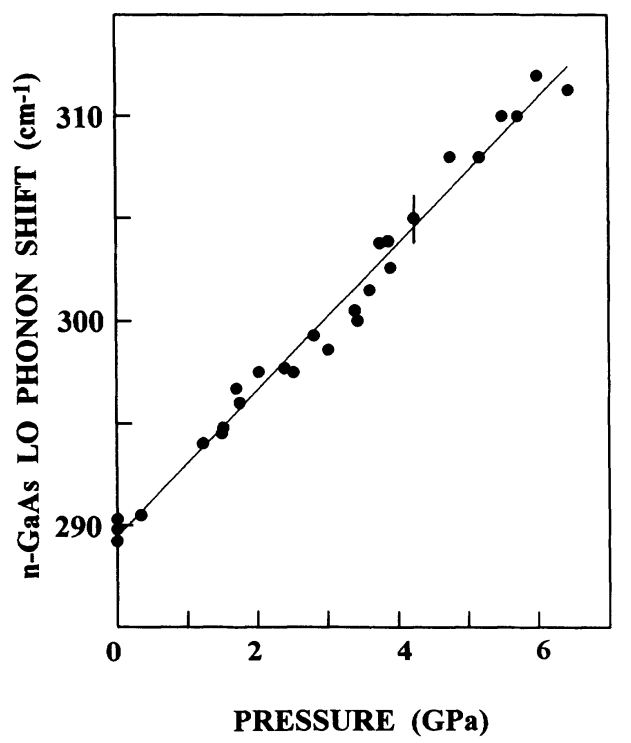

FIG. 2. Data summary of the LO phonon energy versus pressure. The line is a fit to the data with a slope of $3.6 \pm 0.1 \mathrm{~cm}^{-1} / \mathrm{GPa}$, which is the same as the LO phonon pressure coefficient in bulk GaAs. 


$$
\nu(P)=289.4(4)+3.6(1) P .
$$

The vibrational energy, $\nu$, in Eq. (1) is in $\mathrm{cm}^{-1}$ and the pressure, $P$, is in GPa. The pressure coefficient, $3.6 \pm 0.1 \mathrm{~cm}^{-1} / \mathrm{GPa}$ agrees reasonably well with measurements of the pressure coefficient of the LO phonon in bulk GaAs (Ref. 17). This result is interesting since it confirms that the bulk modulus, which originates directly from bond deformation, retains its macroscopic nature even for these small (average length dimension of $5.5 \mathrm{~nm}$ ) crystals. The absence of a size effect at this level is in stark contrast to both optical ${ }^{18-20}$ and vibrational ${ }^{2,9,15}$ properties which are both strongly influenced by finite size at this level. Evidently, the local atomic structure is not altered from the bulk crystal for nanocrystals of this size. This makes sense when viewed from the perspective that there are on the order of $10^{4}$ atoms in a typical nanocrystal.

\section{THE EFFECT OF PRESSURE ON THE $47 \mathrm{~cm}^{-1}$ RESONANT MODE}

In Fig. 3 we show a data summary of the effect of hydrostatic pressure on the resonant mode at $47 \mathrm{~cm}^{-1}$ between 0 and $4 \mathrm{GPa}$. These data points were inherently difficult to obtain, given the close proximity with the intense laser excitation and the associated complications in working with the diamond-anvil cell. After accounting for these factors, we feel the data is very well behaved. The best fit gives a pressure dependence for the low-frequency resonant mode:

$$
\nu(P)=46.7(3)-0.07(15) P,
$$

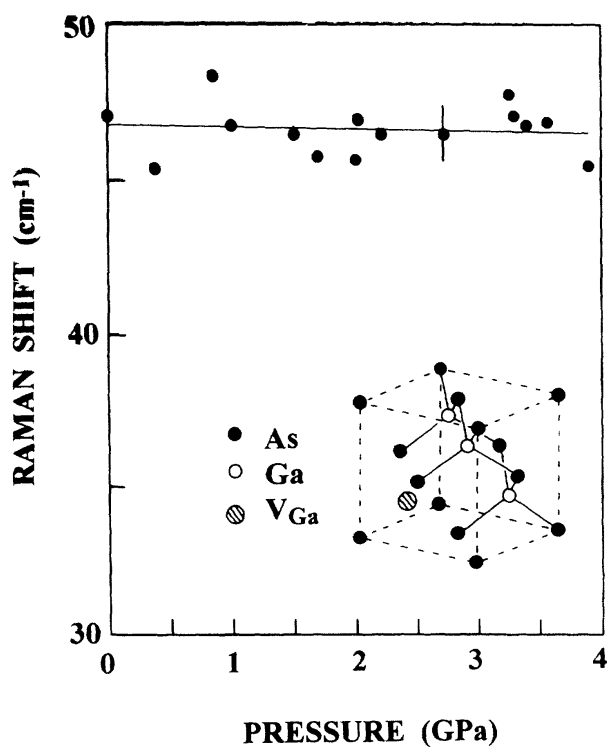

FIG. 3. Data summary of the $47 \mathrm{~cm}^{-1}$ band shown in Fig. 1 versus pressure. The linear fit has a pressure coefficient of $-0.07 \pm 0.15 \mathrm{~cm}^{-1} / \mathrm{GPa}$, supporting the interpretation that this band-resonant mode stems from vacancy point defects, as shown in the inset. where, again, $\nu$ is in $\mathrm{cm}^{-1}$ and $P$ is in GPa. The pressure coefficient given by this equation, $-0.07 \pm$ $0.15 \mathrm{~cm}^{-1} / \mathrm{GPa}$, is close to zero. For comparison, the pressure coefficients of the lowest-energy $\left(60 \mathrm{~cm}^{-1}\right.$, Ref. 21) TA phonon at the $L$ critical point (TA(L)) of the bulk-GaAs Brillouin zone shifts at the much larger rate of $-1.2 \mathrm{~cm}^{-1} / \mathrm{GPa}^{6}{ }^{6}$ Measurements in GaAs/AlAs superlattices, with Raman-active TA folded phonons also yield a larger pressure coefficient (a $29 \mathrm{~cm}^{-1}$ folded TA feature shifts at a rate of $\left.-0.73 \pm 0.14 \mathrm{~cm}^{-1} / \mathrm{GPa}\right) .{ }^{7}$ Folded LA phonons (near $40 \mathrm{~cm}^{-1}$ ) in GaAs/AlAs superlattices exhibit relatively large, positive pressure shifts (approximately $0.6 \mathrm{~cm}^{-1} / \mathrm{GPa}$ for this case).$^{8}$ We conclude that the resonant mode studied here does not stem simply from disorder activated acoustic phonons and is not related to any vibration in ideal GaAs crystals.

\section{DISCUSSION AND CONCLUSIONS}

Our high pressure data support the interpretation that this resonant band originates from a localized defect which is present in high concentrations. The prime candidate is the gallium vacancy (inset of Fig. 3), as discussed in Ref. 3. In Ref. 2 it was argued that point-defect related vibrations, which were degenerate with bulk GaAs acoustic vibrations, would not be observable. This was believed to be the case because motion relevant to the point defect would rapidly degenerate into motion of the surrounding lattice, providing no discrete band. This is opposite to the case for light impurities which produce local vibrational modes. Instead, defect assisted acoustic modes were believed to be the likely origin of the observed Raman mode, for which a measurable negative pressure shift would be expected. The present results argue against this analysis and support the identification of the $47 \mathrm{~cm}^{-1}$ band as the gallium vacancy.

The near-zero pressure coefficient has interesting implications when the separation distance between arsenic atoms is considered under pressure. Evidently, the pressure induced volume deformation (up to $4 \mathrm{GPa}$ ) does not influence the bond lengths to the next nearest neighbors of $V_{\mathrm{Ga}}$. Instead, the contraction is mostly taken up by the arsenic tetrahedron which defines the vacancy. The GaAs crystalline bulk modulus has been shown in Sec. III to remain accurate in these $5.5 \mathrm{~nm}$ crystallites when subjected to these pressures. Pressure will have a different effect on a vacancy. We estimate the volume change of the gallium vacancy. Using $1 / B_{0}=75.4 \mathrm{GPa},{ }^{22}$ an arsenic tetrahedron surrounding a gallium atom (no defect) contracts by $\Delta V / V_{0} \approx 5.3 \%$ at $4 \mathrm{GPa}$. In the $V_{\mathrm{Ga}}$ case, bond lengths to the second-nearest-neighbors of vacancy do not diminish, whereas the gallium second-nearest-neighbor shell decreases in relative volume by about the same $5.3 \%$ fraction above. This implies that the As atom tetrahedron, which defines a spherical vacancy volume, contracts by $\Delta V / V_{0} \approx 8.6 \%$, which is considerably larger than in the perfect crystal case. The initial straight-line separation between the As atoms under ambient conditions in the no-defect case is $3.995 \AA$. This separation decreases to $3.924 \AA$ when increasing the pressure to $4 \mathrm{GPa}$ in an ideal 
crystal. Since $V_{\mathrm{Ga}}$ is an acceptor state, it is expected that the $V_{\mathrm{Ga}}$ state of the defect would expand slightly, when compared with the perfect crystal under ambient pressure. The magnitude of this expansion is unknown, and will depend on the charge state of the defect. Ignoring this effect, this same As-As separation decreases to 3.879 $\AA$ over the same pressure range when a gallium atom is missing. The fact that the resonant vibrations remain virtually unaffected, while the As atoms surrounding a Ga vacancy are pushed together under hydrostatic pressure, indicates that the interaction between the As atoms must be nearly independent of their separation at these distances.

Spectroscopic signatures from the highly studied $V_{\mathrm{Z}_{\mathrm{n}}}$ native point defects in $\mathrm{ZnSe}$ have been published. ${ }^{23}$ They discuss the electronic eigenstates associated with the $V_{\mathrm{Zn}}^{2-}, V_{\mathrm{Zn}}^{-}$, and $V_{\mathrm{Zn}}^{0}$ possible charge configurations of this point defect. In our case, we are most interested in the $V_{\mathrm{Ga}}^{-}$and $V_{\mathrm{Ga}}^{0}$ charge states of the defect. The latter is particularly relevant since we work at room temperature and under intense laser excitation, which would both tend to ionize the acceptor. We cannot infer any definite ordering to the energies of the orbitals which are split due to the crystal field-we do not have sufficient spectroscopic information to deduce this ordering as was accomphished in Ref. 23. The resonance profile seen in Ref. 2 may be a consequence of the conversion (ionization) from one state to another required to place the $V_{\mathrm{Ga}}$ defect in the state necessary for observing a breathing mode.

In conclusion, the use of hydrostatic pressure to examine the various possible origins of the resonant mode in disordered GaAs has proven to be an effective method for studying Raman-active vibrations. Our results support the interpretation of this band as stemming from a localized defect, possibly the $V_{\mathrm{Ga}}$ breathing mode. Clearly, our experiments provide needed input and underscore the necessity for refining theoretical efforts to describe the electronic and vibrational properties of this and similar relevant defects.

\section{ACKNOWLEDGMENTS}

The ion implanted GaAs was provided by Texas Instruments Central Research Laboratory. The authors would like to thank S. K. Estreicher for a critical reading of this manuscript.
${ }^{1}$ A preliminary version of this work was published by $M$. Holtz, T. Sauncy, and R. Zallen, in Proceedings of the Joint AIRAPT/APS Conference on High Pressure Science and Technology, edited by S. C. Schmidt, J. W. Shaner, G. A. Samara, and M. Ross (AIP, New York, 1994), p. 617.

${ }^{2}$ M. Holtz, R. Zallen, and O. Brafman, Phys. Rev. B 37, 2737 (1988); 38, 6097 (1988).

${ }^{3}$ R.S. Berg, N. Mavalvala, T. Steinberg, and F.W. Smith, J. Electron. Mater. 19, 1323 (1990).

${ }^{4}$ L.P. Avakyants, E.D. Obrastsova, and V.S. Gorelik, J. Mol. Struct. 219, 141 (1990).

${ }^{5}$ D.N. Talwar, M.O. Manasreh, C.E. Stutz, R. Kaspi, and K.R. Evans, J. Electron. Mater. 22, 1445 (1993).

${ }^{6}$ R. Trommer, H. Muller, M. Cardona, and P. Vogl, Phys. Rev. B 21, 4869 (1980).

${ }^{7}$ M. Holtz, T. Sauncy, K. Ploog, and L. Tapfer, Phys. Rev. B 48, 11057 (1993).

${ }^{8}$ M. Holtz, K. Syassen, and K. Ploog, Phys. Rev. B 40, 2988 (1989).

${ }^{9}$ M. Holtz, R. Zallen, O. Brafman, and S. Matteson, Phys. Rev. B 37, 4609 (1988).

${ }^{10}$ D.E. Aspnes and A.A. Studna, Phys. Rev. B 27, 985 (1983).

${ }^{11}$ B.A. Weinstein and R. Zallen, in Light Scattering in Solids, edited by M. Cardona and G. Güntherodt (Springer, Berlin, 1984), p. 463.

${ }^{12}$ G.J. Piermarini, S. Block, J.D. Barnett, and R.A. Forman, J. Appl. Phys. 46, 2779 (1975).

${ }^{13}$ M. Holtz, S.A. Solin, and T.J. Pinnavaia, Phys. Rev. B 48, 13312 (1993).

${ }^{14}$ T. Dallas, M. Holtz, H. Ahn, and M.C. Downer, Phys. Rev. B 49, 796 (1994).

${ }^{15}$ K.K. Tiong, P.M. Amirtharaj, F.H. Pollak, and D.E. Aspnes, Appl. Phys. Lett. 44, 122 (1983).

${ }^{16}$ R. Zallen, M. Holtz, A. E. Geissberger, R. Sadler, W. Paul, and M.-L. Theye, J. Non-Cryst. Solids 114, 795 (1989).

${ }^{17}$ U.D. Venkateswaran, L.J. Cui, B.A. Weinstein, and F.A. Chambers, Phys. Rev. B 45, 9237 (1992).

${ }^{18}$ D.E. Aspnes, S.M. Kelso, C.G. Lynch, and D.W. Lynch, Phys. Rev. Lett. 48, 1863 (1982).

19 J.B. Theeten and M. Erman, J. Vac. Sci. Technol. 20, 471 (1982).

${ }^{20}$ G.F. Feng and R. Zallen, Phys. Rev. B 40, 1064 (1989).

${ }^{21}$ J.L.T. Waugh and G. Dolling, Phys. Rev. 132, 2410 (1963).

${ }^{22}$ H.J. McSkimin, A. Jayaraman, and P. Andreatch, J. Appl. Phys. 38, 2362 (1967).

${ }^{23}$ See Duk Y. Jeon, H.P. Gislason, and G.D. Watkins, Phys. Rev. B 48, 7872 (1993), and references therein. 